\title{
Antimalarial drugs inhibit calcium-dependent backward swimming and calcium currents in Paramecium calkinsi
}

\author{
S.R. Barry ${ }^{1,3, *}$, J. Bernal ${ }^{2,3}$ \\ ${ }^{1}$ Department of Physical Medicine \& Rehabilitation, University Hospital Rm 1D204, University of Michigan, Ann Arbor, \\ MI 48109-0042, USA \\ ${ }^{2}$ Departamento de Fisiologia y Farmacologia, Universidad Autonoma de Aguascalientes, Aguascalientes, Ags, CP 20100, Mexico \\ ${ }^{3}$ Marine Biological Laboratory, Woods Hole, MA 02543, USA
}

Accepted: 29 December 1992

\begin{abstract}
The antimalarial drugs, quinacrine, chloroquine, quinine, primaquine, and mefloquine, share structural similarities with W-7, a compound that inhibits calcium-dependent backward swimming and calcium currents in Paramecium. Therefore, we tested whether antimalarial drugs also inhibit backward swimming and calcium currents in P. calkinsi. When the Paramecium is depolarized in high potassium medium, voltage-dependent calcium channels in the ciliary membrane open causing the cell to swim backward for 30 to $70 \mathrm{~s}$. Application of calcium channel inhibitors, such as W-7, reduce the duration of backward swimming. In $0.05 \mathrm{~m} M$ calcium, quinacrine, mefloquine, quinine, chloroquine, primaquine and $\mathrm{W}-7$ all reduced the duration of backward swimming. These effects were seen in sodium-containing and sodium-free high potassium solutions as well as sodium-free depolarizing solutions containing potassium channel blockers. In these low calcium solutions, backward swimming was inhibited by $50 \%$ at concentrations ranging from $100 \mathrm{nM}$ to $30 \mu M$. At higher calcium concentrations $(1 \mathrm{mM}$ or $15 \mathrm{mM})$, the effects of the antimalarials and $\mathrm{W}-7$ were reduced. The effects of quinacrine and $\mathrm{W}-7$ were tested directly on calcium currents using the two microelectrode voltage clamp technique. In $15 \mathrm{~m} M$ calcium, $100 \mu M$ quinacrine and 100 $\mu M \mathrm{~W}-7$ reduced the peak calcium current by $51 \%$ and $42 \%$, respectively. Thus, antimalarial drugs reduce calcium currents in Paramecium calkinsi.
\end{abstract}

Key words: Paramecium - Antimalarial drugs - Calcium currents - Quinacrine - Calcium channel blockers

\section{Introduction}

The mechanism of action of the antimalarial drugs, quinine, quinacrine, chloroquine, primaquine and meflo-

* Current address: Dept. of Biological Sciences, Mount Holyoke College, South Hadley, MA 01075, USA

Correspondence to: S.R. Barry quine, is not completely understood. These drugs share structural similarities with compounds that inhibit ionic currents. In particular, the antimalarials resemble W-7 (N-(6-aminohexyl)-5-chloro-1-naphthalenesulfonamide) (Fig. 1), a compound that inhibits calcium-dependent backward swimming and calcium currents in $P$. caudatum and $P$. tetraurelia but does not affect calcium currents in vertebrate nerve and muscle cells (Hennessey and Kung 1984; Ehrlich et al. 1988). Therefore, we hypothesized that antimalarial drugs, like W-7, inhibit calcium-dependent backward swimming and calcium currents in Paramecium.

Paramecium (P. tetraurelia, P. caudatum, and P. calkinsi) possess an excitable cell membrane (Eckert and Brehm 1979; Saimi et al. 1988; Preston 1990). A brief depolarization of the cell membrane produces a graded action potential whose rising phase results from activation of voltage-dependent calcium channels and whose repolarizing phase results from inactivation of calcium channels and activation of one or more classes of potassium channels.
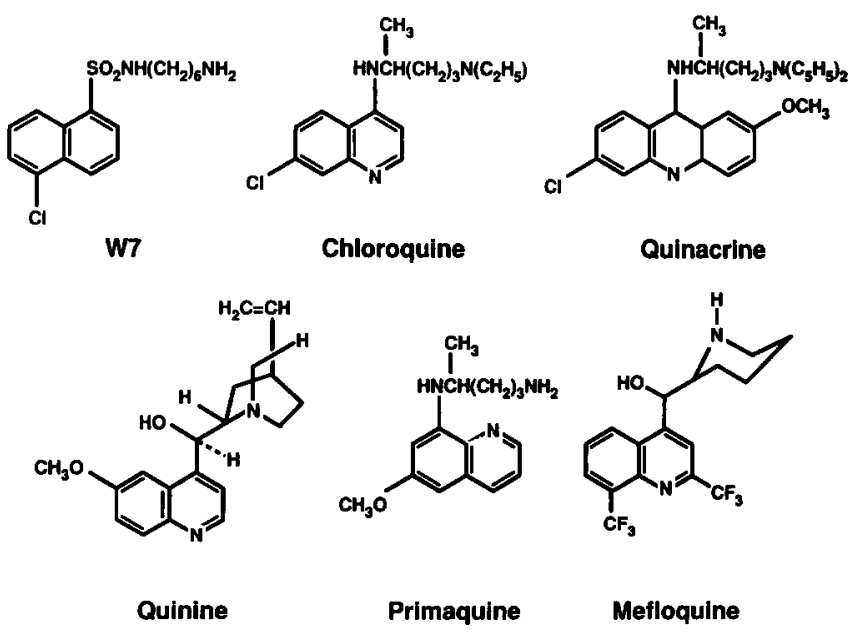

Fig. 1. Antimalarial drugs are similar in structure to W-7, a calcium channel blocker in Paramecium. Quinacrine, chloroquine, and W-7 possess a hydrophobic ring structure, a chloride residue and a long alkyl side chain terminating in an amino group 
Table 1. Composition of nutrient and recording media

\begin{tabular}{|c|c|c|c|c|c|c|c|c|}
\hline & Sol. 1 & Sol. 2 & Sol. 3 & Sol. 4 & Sol. 5 & Sol. 6 & Sol. 7 & Sol. 8 \\
\hline $\mathrm{NaCl}$ & 125 & 125 & 62.5 & 62.5 & - & 145 & - & - \\
\hline NaCitrate & 1 & - & - & - & - & - & - & - \\
\hline $\mathrm{CaCl}_{2}$ & 5 & $0.05,1$, or 15 & $0.05,1$, or 15 & 0.05 & 0.05 & 0.05 & 0.05 & 15 \\
\hline $\mathrm{MgCl}_{2}$ & 10 & - & - & - & - & - & - & - \\
\hline MOPS & 10 & 10 & 10 & 10 & 10 & 10 & 10 & 10 \\
\hline TEA-Cl & - & - & - & - & - & - & 125 & 125 \\
\hline $\mathrm{CsCl}$ & - & - & - & - & - & - & 10 & 10 \\
\hline 4-aminopyridine & - & - & - & - & - & - & 5 & 5 \\
\hline 3,4-diaminopyridine & - & - & - & - & - & - & 5 & 5 \\
\hline
\end{tabular}

All concentrations are in $\mathrm{mM} . \mathrm{pH}$ is adjusted to $7.2-7.4$.

Sol. 1 = nutrient media for culturing $P$. calkinsi.

Sol.'s 2 and 3 = resting and depolarizing media for backward swimming experiments in sodium-containing, high potassium solution, respectively.

Sol.'s 4 and $5=$ resting and depolarizing media for backward swim-

Ionic channels regulate the swimming behavior of paramecia (Eckert 1972; Eckert and Brehm 1979; Machemer 1989). Paramecia swim by beating the thousands of cilia that are uniformly distributed over the surface of the cell. Activation of the voltage-sensitive calcium channels and the resultant calcium influx cause the cilia to reverse the direction of their power stroke. The reversal in the ciliary beat causes the Paramecium to back up and reorient its swimming direction. Thus, the swimming behavior of Paramecium reflects the activity of ionic channels in the cell membrane.

When the Paramecium is transferred to a high potassium solution, the cell is depolarized and the Paramecium swims backward for many seconds. The duration of backward swimming in high potassium solutions can be used as an indicator of calcium channel activity (Hennessey and Kung 1984). Drugs, such as W-7, that reduce the duration of backward swimming inhibit calcium currents measured from voltage-clamped cells (Hennessey and Kung 1984).

We have observed the effects of W-7 and antimalarial drugs on calcium channels in the marine Paramecium, $P$. calkinsi, by testing the actions of the drugs on calciumdependent swimming behavior and calcium currents. Since the effects in P. caudatum of other calcium channel inhibitors vary with the extracellular calcium levels (Gustin and Hennessey 1988), we have observed the actions of the antimalarials on backward swimming at different extracellular calcium concentrations. The antimalarials reduced the duration of backward swimming in high potassium solutions, particularly at low extracellular calcium concentrations. Moreover, the voltage-dependent calcium current in $P$. calkinsi was decreased by micromolar concentrations of quinacrine and W-7. These data show that quinacrine and other antimalarial drugs can modulate calcium-dependent swimming and calcium currents in $P$. calkinsi. Preliminary reports of these studies have been published (Barry et al. 1988, 1990, 1991). ming experiments in sodium-free, high potassium solution, respectively.

Sol.'s 6 and $7=$ resting and depolarizing solutions for backward swimming experiments in TEA solution, respectively.

Sol. $8=$ recording medium for electrophysiological experiments.

\section{Materials and methods}

\section{Preparation}

Paramecium calkinsi, a marine ciliate, were cultured at room temperature $\left(20-23^{\circ} \mathrm{C}\right)$ in a nutrient medium that was found to promote excellent cell growth (Solution 1, Table 1). This medium contained stigmasterol $(5 \mathrm{mg} / \mathrm{l})$ and was buffered with MOPS . Cells were fed with Enterobacter aerogenes every $48 \mathrm{~h}$.

\section{Behavioral assays}

Backward swimming in high potassium, sodium medium. P. calkinsi were transferred from the culture medium to a "resting solution" containing (in $\mathrm{mM}$ ): $\mathrm{NaCl}(125)$, MOPS (10), and $\mathrm{CaCl}_{2}(0.05,1$, or 15) (Solution 2, Table 1) and varying concentrations of $W-7$ or the antimalarial drugs. After $5 \mathrm{~min}$, approximately 10 paramecia were transferred from the resting solution to a depolarizing solution which was identical to the resting solution except that it contained $62.5 \mathrm{mM} \mathrm{KCl}$ and $62.5 \mathrm{mM} \mathrm{NaCl}$ (Solution 3, Table 1). In the high potassium solution without drugs, the paramecia swam backward for 30 to $70 \mathrm{~s}$, spun in place about their posterior end and swam forward in tight circles before resuming normal forward swimming. As defined by Machemer and Sugino (1989), the time period from transfer of the paramecia to the high potassium solution until stationary spinning was measured as the duration of backward swimming. The duration of backward swimming of at least 10 different paramecia was measured for control conditions and for each concentration of each drug tested. Concentrations at which half-maximal effects occurred were estimated from the concentration-response curves.

Backward swimming in high potassium, sodium-free medium. Backward swimming was also measured in a depolarizing solution containing no sodium. For these experiments, the resting solution contained $62.5 \mathrm{mM} \mathrm{NaCl}, 0.05 \mathrm{mM} \mathrm{CaCl}$, and $10 \mathrm{mM}$ MOPS (Solution 4, Table 1) while the depolarizing solution contained $62.5 \mathrm{mM}$ $\mathrm{KCl}, 0.05 \mathrm{mM} \mathrm{CaCl}$, and $10 \mathrm{mM}$ MOPS (Solution 5, Table 1). The low calcium concentration was chosen for these experiments because the effects on backward swimming of the antimalarials were most potent in high potassum, sodium solutions containing 0.05 $\mathrm{mM} \mathrm{CaCl}$. 
Backward swimming in TEA medium. A final series of experiments were performed in a depolarizing medium containing no sodium or potassium and containing potassium channel blockers. For these experiments, the paramecia were transferred from a resting solution containing $145 \mathrm{mM} \mathrm{NaCl}, 0.05 \mathrm{mM} \mathrm{CaCl}$, and $10 \mathrm{mM}$ MOPS (Solution 6, Table 1) to a "TEA solution" containing $125 \mathrm{mM}$ tetraethylammonium chloride (TeaCl), $10 \mathrm{mM} \mathrm{CsCl,} 5 \mathrm{mM} 4-$ aminopyridine, $5 \mathrm{~m} M$ 3,4-diaminopyridine, $0.05 \mathrm{mM} \mathrm{CaCl}$, and 10 $\mathrm{m} M$ MOPS (Solution 7, Table 1). With the exception of the low calcium concentration, this solution was identical to the recording medium used in the electrophysiological experiments. Transfer of the paramecia from the resting to the TEA solution depolarized the cells as a result of inhibition of potassium channels. In this medium, the cells swam backward for about 25 to $29 \mathrm{~s}$. After the initial bout of backward swimming, the paramecia swam forward with frequent brief bouts of backward swimming. The duration of the initial period of backward swimming was measured and compared for paramecia in the absence and presence of $\mathrm{W}-7$ and the antimalarial drugs.

\section{Electrophysiological recordings}

In order to impale swimming paramecia with microelectrodes, the cells must be trapped first. Previous studies on fresh water paramecia have used the "hanging drop" method to trap the swimming cells (Naitoh and Eckert 1972). In this procedure, the solution surrounding the Paramecium is allowed to evaporate until the drop is approximately the same size as the cell. Since we used marine paramecia in this study, the recording solution contained a higher concentration of ions than in studies using fresh water paramecia. As a result, evaporation of the recording media to produce a hanging drop resulted in a salt concentration in the drop that was prohibitively high. Thus, a second method using agarose was devised to trap the paramecia.

Briefly, cells from the culture media (Solution 1, Table 1) were harvested and washed twice with culture medium. A small volume $(<25 \mu l)$ of solution containing concentrated paramecia was set on the recording chamber. After the drop was slightly evaporated ( 3 to $5 \mathrm{~min}$ ), an agarose solution $(5 \mathrm{mg}$ agarose/dissolved in $1 \mathrm{ml}$ of recording medium) was added to the paramecia. After the solution was mixed, the chamber was placed in the freezer $\left(-20^{\circ} \mathrm{C}\right)$ for $30 \mathrm{~s}$. In the freezer, the agarose cooled into a jello-like matrix and trapped the paramecia in a volume approximately the same size as the cell. Paramecia were viable in the agarose solution for more than $24 \mathrm{~h}$.

Once the paramecia were trapped in the agarose, the preparation was placed on the stage of a Nikon Diaphot inverted microscope and perfused with a sodium-free, magnesium-free recording medium containing $15 \mathrm{mM} \mathrm{CaCl}$ and potassium channel blockers (Solution 8, Table 1). Stock solutions of $\mathrm{W}-7$ and quinacrine were made up in recording medium or de-ionized water, and the drugs, at concentrations ranging from 0.1 to $1 \mathrm{mM}$, were introduced to the preparation by perfusion. Experiments were performed at room temperature $\left(20-23^{\circ} \mathrm{C}\right)$. Previous experiments in the laboratory have indicated that compounds in the perfusion medium readily diffused through the agarose layer to reach the cells. For example, application of potassium channel blockers broadened the action potential and reduced all outward currents within seconds of application to the preparation (data not shown).

The paramecia were impaled with two microelectrodes and voltage-clamped using a Dagan 8500 amplifier (Dagan Corporation, Mineapolis, MN). Microelectrodes were filled with $0.3 \mathrm{M}$ $\mathrm{CsCl}, 5 \mathrm{mM} \mathrm{MgCl}$, and $10 \mathrm{mM}$ MOPS ( $\mathrm{pH} \mathrm{7.0)}$ and bevelled to a resistance of 5 to $20 \mathrm{M} \Omega$ with a model BV-10, K.T. Brown Type Micropipette Beveler (Sutter Instrument Co., San Novato, CA.). The addition of the $\mathrm{MgCl}_{2}$ to the microelectrode filling solution increased the viability of the impaled cells. The paramecia were voltage-clamped at the resting potential of the cell $(-20$ to $-40 \mathrm{mV})$. The membrane potential was changed with 50 to $70 \mathrm{~ms}$ depolarizing voltage steps at $10 \mathrm{mV}$ increments, and the current responses to these voltage steps was measured in the presence and absence of $\mathrm{W}-7$ and quinacrine. Linear leakage currents were extrapolated from the response to a $10 \mathrm{mV}$ hyperpolarizing step and then subtracted from the current traces. The stimulation protocol was delivered and the data analyzed using pClamp software (version 5.0, Axon Instruments) installed on an IBM AT clone.

\section{Chemical solutions}

Quinacrine, chloroquine, primaquine, quinine, W-7, tetraethylammonium chloride, 4-aminopyridine, 3,4, diaminopyridine, and cesium chloride were purchased from Sigma Chemical Co. Mefloquine was generously provided by Hoffmann-La Roche, Inc.. Stock solutions for W-7 and the antimalarial drugs were prepared at 10 $\mathrm{m} M$ concentrations dissolved in de-ionized water or at $1 \mathrm{~m} M$ concentrations dissolved directly in the experimental solutions.

\section{Statistics}

Significant differences between mean values were tested using a two-sample, two-tailed Student's $t$-test. Mean values were judged to be significantly different if the $P$ value was less than 0.05 . All values in the paper are represented as mean \pm standard error of the mean.

\section{Results}

\section{Effects of antimalarial drugs on backward swimming}

The effects of the antimalarials on backward swimming were tested under several different conditions including high potassium, sodium medium containing $0.05 \mathrm{mM}, 1$ $\mathrm{mM}$, and $15 \mathrm{mMCaCl}$, high potassium, sodium-free medium containing $0.05 \mathrm{mM} \mathrm{CaCl}$, and TEA medium containing $0.05 \mathrm{mM}$ and $15 \mathrm{mM} \mathrm{CaCl}$.

In high potassium, sodium solutions containing $1 \mathrm{mM}$ $\mathrm{CaCl}_{2}$, P. calkinsi swam backward for approximately $70 \mathrm{~s}$ (Fig. 2). A decrease in $\mathrm{CaCl}_{2}$ concentration to $0.05 \mathrm{mM}$

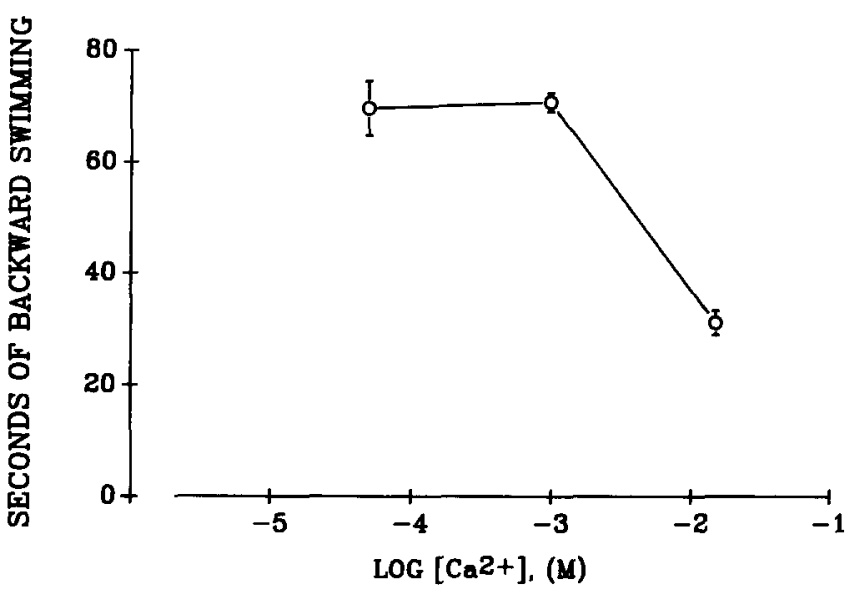

Fig. 2. The duration of backward swimming was measured in high potassium, sodium medium containing $0.05 \mathrm{mM} \mathrm{CaCl}, 1 \mathrm{mM} \mathrm{Ca-}$ $\mathrm{Cl}_{2}$ and $15 \mathrm{mM} \mathrm{CaCl}$. There was no significant difference in the duration of backward swimming in 0.05 and $1 \mathrm{mM} \mathrm{CaCl}$. However, backward swimming was reduced by $56 \pm 5 \%$ in $15 \mathrm{mM} \mathrm{CaCl}_{2}$. Data represent the mean and S.E.M. of the duration of backward swimming for 10 to 21 paramecia 


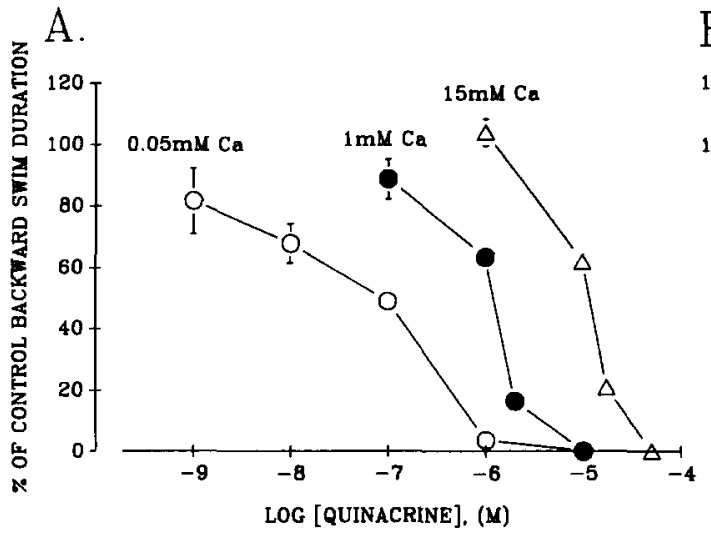

Fig. 3. Quinacrine (A) and W-7 (B) reduced the duration of backward swimming in $P$. calkinsi. The drugs were more potent in 0.05 $\mathrm{m} M \mathrm{CaCl}_{2}(\mathrm{O})$ than in $1 \mathrm{~m} M \mathrm{CaCl}_{2}(\bigcirc)$ or $15 \mathrm{mM} \mathrm{CaCl}(\triangle)$. Significant effects of quinacrine were seen at $10 \mathrm{nM}$ in $0.05 \mathrm{mM}$ $\mathrm{CaCl}_{2}$, at $1 \mu M$ in $1 \mathrm{mMCaCl}$, and at $10 \mu M$ in $15 \mathrm{mM} \mathrm{CaCl}$.

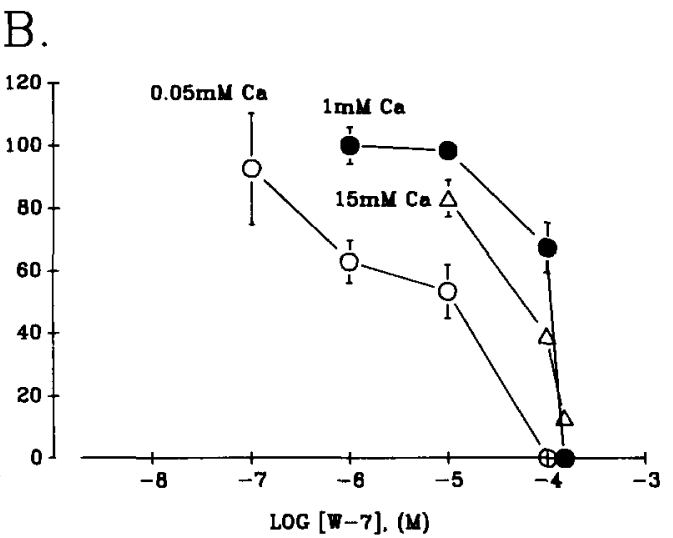

Significant effects of $\mathrm{W}-7$ were seen at $1 \mu M$ in $0.05 \mathrm{mM} \mathrm{CaCl}$ and at $100 \mu M$ in $1 \mathrm{mM}$ and $15 \mathrm{mM} \mathrm{CaCl}{ }_{2}$. Data represent the mean and S.E.M of the duration of backward swimming for 10 to 14 paramecia. On some points, error bars are not seen because the bars are smaller than the symbol size

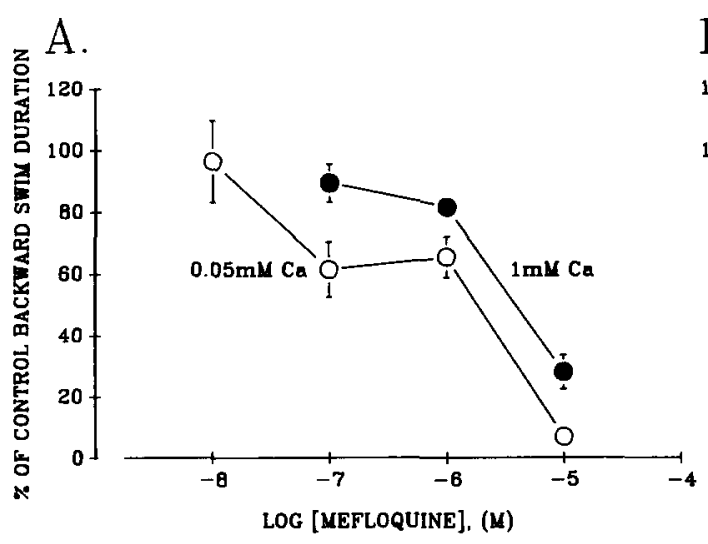

Fig. 4. Mefloquine (A) and quinine (B) reduced the duration of backward swimming in $P$. calkinsi. The drugs were more potent in $0.05 \mathrm{mM} \mathrm{CaCl}_{2}(\mathrm{O})$ than in $1 \mathrm{mM} \mathrm{CaCl}(\mathbf{O})$. Significant effects of mefloquine were seen at $100 \mathrm{n} M$ in $0.05 \mathrm{mM} \mathrm{CaCl}$ and at $1 \mu M$ in $1 \mathrm{mM} \mathrm{CaCl}{ }_{2}$. Significant effects of quinine were seen at $1 \mu M$ in 0.05

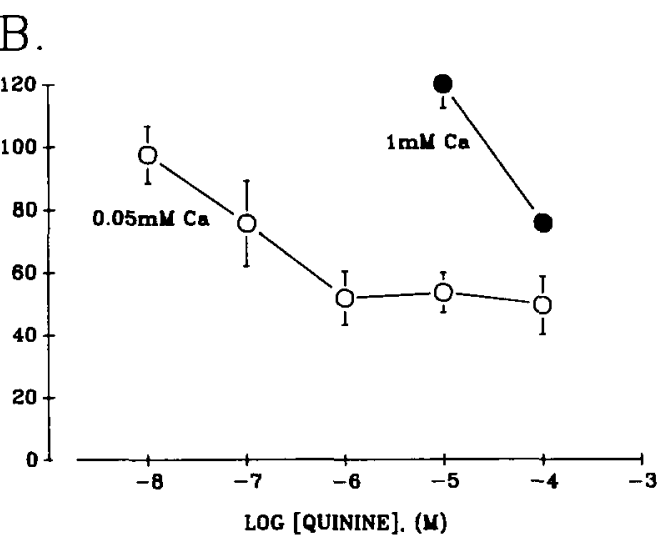

$\mathrm{m} M \mathrm{CaCl}_{2}$ and at $100 \mu M$ in $1 \mathrm{~m} M \mathrm{CaCl}_{2}$. Data represent the mean and S.E.M of the duration of backward swimming for 10 to 19 paramecia. On some points, error bars are not seen because the bars are smaller than the symbol size produced little or no effect on the average duration of backward swimming although, in the low calcium solution, there was more variability from cell to cell in the backward swimming duration. In contrast, an increase in $\mathrm{CaCl}_{2}$ concentration from $1 \mathrm{~m} M$ to $15 \mathrm{mM}$ significantly decreased the duration of backward swimming by $56 \pm 5 \%(70.8 \pm 1.7 \mathrm{~ms}$ in $1 \mathrm{mM} \mathrm{CaCl} ; 31.2 \pm 2.2 \mathrm{~s}$ in 15 $\mathrm{m} \bar{M} \mathrm{CaCl}_{2}, n=16$ for $1 \mathrm{~m} M \mathrm{CaCl}_{2}$ and $n=12$ for $15 \mathrm{mM}$ $\mathrm{CaCl}_{2}$ ) (Fig. 2).

High potassium, sodium medium containing $0.05 \mathrm{mM} \mathrm{Ca}$ $\mathrm{Cl}_{2}$. W-7 and the antimalarial drugs depressed the duration of backward swimming in high potassium, sodium solutions containing low calcium concentrations. In 0.05 $\mathrm{mMCaCl}$, quinacrine produced a significant reduction in the duration of backward swimming at a concentration of $10 \mathrm{n} M(39 \pm 4 \mathrm{~s}$ without quinacrine and $27 \pm 4 \mathrm{~s}$ with quinacrine ( $n=10$ for both conditions), reduced backward swimming by $50 \pm 6.5 \%$ at $100 \mathrm{n} M(n=10)$ and almost entirely eliminated backward swimming at $1 \mu M$ (Fig. 3A). All of the drugs reduced backward swimming by $50 \%$ at concentrations of $30 \mu M$ or less (Fig. 3-5). The rank order potency of the drugs in reducing backward swimming in $0.05 \mathrm{mM} \mathrm{CaCl}$ was quinacrine $>$ mefloquine $\geq$ quinine $\geq \mathrm{W}-7>$ chloroquine $>$ primaquine.

High potassium, sodium medium containing $1 \mathrm{mM} \mathrm{CaCl}$. An increase in extracellular $\mathrm{CaCl}_{2}$ to $1 \mathrm{mM}$ in the high potassium, sodium medium reduced the efficacy of the drugs in inhibiting backward swimming. Effects of quinacrine, mefloquine, quinine, W-7 and choroquine were observed at 2 to 100 -fold higher concentrations in 1 $\mathrm{m} M$ as opposed to $0.05 \mathrm{mM} \mathrm{CaCl}$ (Fig. 3-5). In $1 \mathrm{mM}$ $\mathrm{CaCl}_{2}$, primaquine was no longer effective in inhibiting backward swimming at concentrations up to $300 \mu M$ 


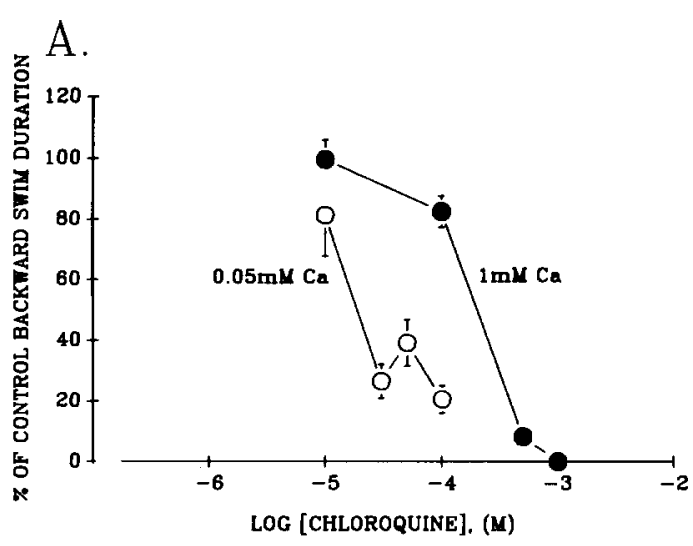

Fig. 5. Chloroquine (A) and primaquine (B) reduced the duration of backward swimming in $P$. calkinsi. The drugs were more potent in $0.05 \mathrm{mM} \mathrm{CaCl}(O)$ than in $1 \mathrm{mM} \mathrm{CaCl}(O)$. Significant effects of chloroquine were seen at $30 \mu \mathrm{M}$ in $0.05 \mathrm{mM} \mathrm{CaCl}_{2}$ and at $100 \mu M$ in $1 \mathrm{~m} M \mathrm{CaCl}_{2}$. Significant effects of primaquine were seen at

B.

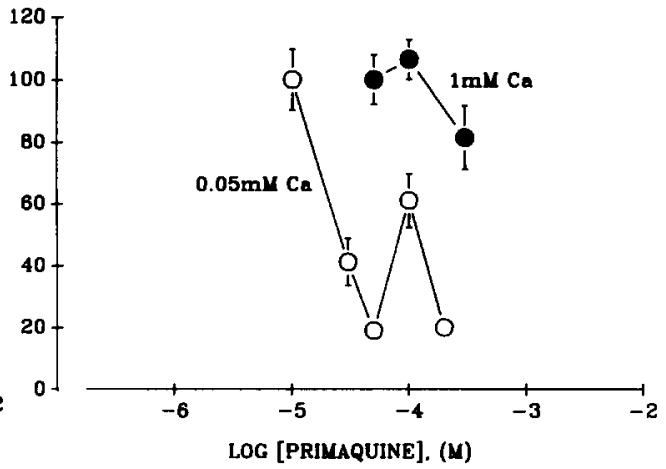

$30 \mu M$ in $0.05 \mathrm{mM} \mathrm{CaCl}_{2}$. No significant effects were seen with primaquine in $1 \mathrm{mM} \mathrm{CaCl}$ at concentrations as high as $300 \mu M$. Data represent the mean and S.E.M of the duration of backward swimming for 10 to 16 paramecia. On some points, error bars are not seen because the bars are smaller than the symbol size

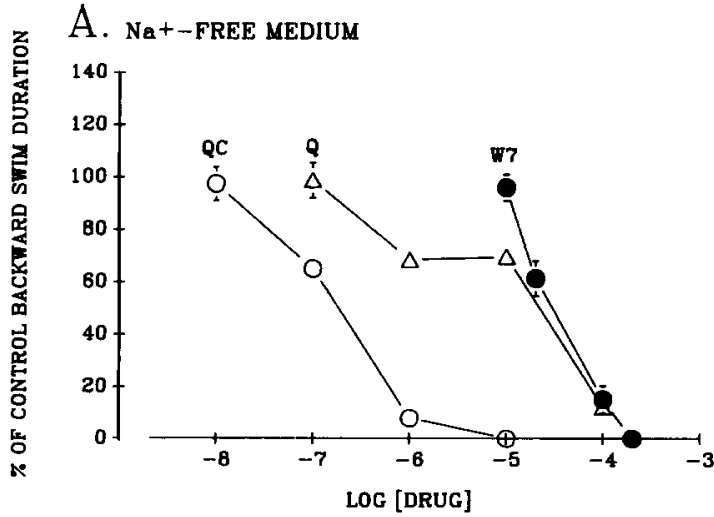

Fig. 6. In part $\mathbf{A}$, quinacrine $(Q C, O)$, quinine $(Q, \triangle)$, and $\mathrm{W}-7$, $(W 7, O)$ and, in part $\mathbf{B}$, chloroquine $(C Q, \bigcirc)$, mefloquine $(M Q, O)$, and primaquine $(P Q, \triangle)$ reduced the duration of backward swimming in high potassium, sodium-free medium, containing $0.05 \mathrm{mM}$ $\mathrm{CaCl}_{2}$. Significant effects were seen for quinacrine at $100 \mathrm{n} M$,

(Fig. 5B). At higher concentrations, the drug was toxic to the paramecia within minutes.

High potassium, sodium medium containing $15 \mathrm{mM} \mathrm{CaCl}$. The effects of quinacrine and W-7 on backward swimming were also tested in depolarizing solutions containing $15 \mathrm{mM} \mathrm{CaCl}{ }_{2}$ since this is the concentration of $\mathrm{CaCl}_{2}$ routinely used in electrophysiological experiments. Effects of quinacrine were seen at approximately $10-$ and 100 -fold higher concentrations in $15 \mathrm{mM} \mathrm{CaCl}_{2}$ than in $1 \mathrm{mM}$ and $0.05 \mathrm{mM} \mathrm{CaCl}{ }_{2}$ respectively (Fig. 3A). In contrast, little difference was seen in the effects of W-7 in $15 \mathrm{mM} \mathrm{CaCl}$ as opposed to $1 \mathrm{mM} \mathrm{CaCl}{ }_{2}$ (Fig. 3B). With the exception of W-7's effects in $15 \mathrm{mM} \mathrm{CaCl}$, the effects of all the drugs increased in potency with decreasing ambient calcium levels.

High potassium, sodium-free medium containing $0.05 \mathrm{mM}$ $\mathrm{CaCl}_{2}$. In fresh-water paramecia ( $P$. tetraurelia), backward swimming experiments are performed in a sodium- free, depolarizing medium. These paramecia possess a calcium-dependent sodium current that prolongs backward swimming in sodium-containing medium (Saimi and Kung 1980). If such a current exists in P. calkinsi, the inhibitory effects of the antimalarials may result from modulation of the calcium-dependent sodium current as opposed to inhibition of the calcium current. Therefore, the effects of W-7 and the antimalarials on backward swimming were studied in depolarizing solutions containing no sodium. Since the effects of the antimalarials in high-potassium, sodium solutions were greatest at low calcium concentrations, the actions of the drugs were examined in a sodium-free depolarizing medium containing $0.05 \mathrm{mM} \mathrm{CaCl}{ }_{2}$. Transfer of the paramecia from the resting solution to the high potassium, sodium-free medium caused the paramecia to swim backward for a period of 74 to $125 \mathrm{~s}$. All of the drugs inhibited backward swimming in the high potassium, sodium-free solutions (Fig. 6). With the exception of quinine, the concentrations that 


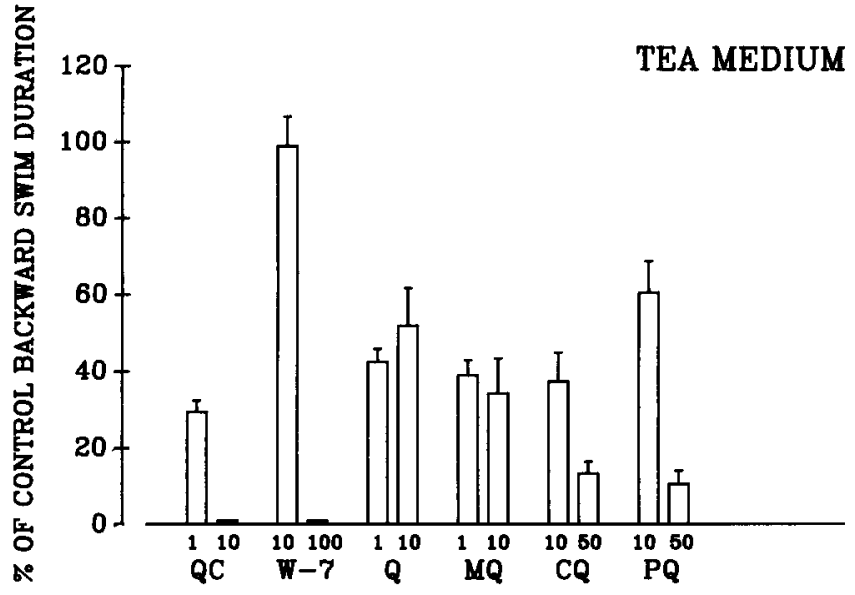

Fig. 7. Quinacrine $(Q C), \mathrm{W}-7(W 7)$, quinine $(Q)$, mefloquine $(M Q)$, chloroquine $(C Q)$, and primaquine $(P Q)$ reduced the duration of backward swimming in TEA medium containing $0.05 \mathrm{mM} \mathrm{CaCl}$. Numbers below the data bars indicate drug concentrations in $\mu M$. All effects are significant except for W-7 at a concentration of $10 \mu M$. Data represent the mean and S.E.M of the duration of backward swimming for 10 to 18 paramecia

produced 70 to $100 \%$ inhibition of backward swimming were similar for the drugs in the absence and presence of sodium. At $100 \mu M$, quinine inhibited backward swimming by only $50 \pm 7 \%(n=16)$ in sodium solutions but by $88 \pm 4 \%(n=10)$ in sodium-free solutions. Moreover, chloroquine depressed backward swimming significantly by $56 \pm 4 \%(n=10)$ at $100 \mathrm{nM}$ in sodium-free medium but had no significant effect until a concentration of 30 $\mu M$ in sodium-containing medium. Thus, the effects of these drugs may be more potent in sodium-free, depolarizing medium.

$T E A$ medium containing $0.05 \mathrm{mM} \mathrm{CaCl}$. The effects of the antimalarials on backward swimming were also tested in a sodium-free, potassium-free solution containing potassium channel blockers (TEA medium). This solution contained the low calcium concentration of $0.05 \mathrm{mM} \mathrm{CaCl} \mathrm{Cl}_{2}$ but was otherwise identical to the recording medium used in the electrophysiological experiments. Transfer of the paramecia to this solution depolarizes the paramecia by inhibition of potassium currents. In the TEA medium, the paramecia undergo an initial bout of backward swimming of 25 to $29 \mathrm{~s}$. Since both sodium and potassium currents are substantially reduced in the TEA medium, inhibitory effects of the antimalarials on backward swimming would strongly suggest that the antimalarials depress backward swimming by inhibition of calcium currents. The effects of W-7 and the antimalarials on backward swimming in the TEA medium were tested at those concentrations that produced a significant reduction in the duration of backward swimming in the low calcium, high potassium solution containing sodium. At these concentrations in TEA medium, all of the drugs significantly reduced the duration of backward swimming with the exception of W-7 at a concentration of $10 \mu M$ (Fig. 7).

TEA medium containing $15 \mathrm{mM} \mathrm{CaCl}$. Finally, the actions of quinacrine and $\mathrm{W}-7$ on backward swimming were tested in TEA medium containing $15 \mathrm{mM} \mathrm{CaCl}$. This medium is identical to the solution used in the voltage clamp experiments described below. Transfer of the paramecia to TEA medium containing $15 \mathrm{mM} \mathrm{CaCl} \mathrm{Ca}_{2}$ caused the paramecia to swim backward for 5 to $31 \mathrm{s.} 10$ $\mu M$ quinacrine reduced significantly the duration of backward swimming by $79 \pm 11 \% \quad(n=16$ for control; $n=10$ for quinacrine) while $100 \mu M$ quinacrine depressed backward swimming by $100 \pm 0 \%$ ( $n=10$ for quinacrine). $100 \mu M \mathrm{~W}-7$ also reduced backward swimming significantly by $89 \pm 5 \%$ ( $n=10$ for both conditions).

\section{Effects of quinacrine and W-7 on calcium currents}

Quinacrine was the most potent modulator of backward swimming in $P$. calkinsi among the antimalarial drugs tested. Moreover, the drug was more effective than W-7 in inhibiting this behavior. To test whether the effects of these drugs on backward swimming were mediated by inhibition of calcium channels, we tested the effects of quinacrine and W-7 on calcium currents in P. calkinsi.

In preliminary experiments, the effects of quinacrine were observed on the calcium action potential (Barry et al. 1988). Paramecia were placed in a sodium- and magnesium-free recording medium containing potassium channel blockers (Solution 8, Table 1). Under these conditions, the rising phase of the action potential resulted solely from calcium influx while the repolarizing phase resulted from calcium channel inactivation. Quinacrine reversibly reduced the amplitude and duration of the calcium action potential (data not shown). To test whether this effect of quinacrine was due to calcium channel inhibition, calcium currents were recorded from Paramecium using the two microelectrode voltage clamp technique.

$P$. calkinsi were voltage-clamped in the sodium-free, magnesium-free recording medium containing potasssium channel blockers. Under these conditions, depolarizing voltage steps of less than $80 \mathrm{mV}$ from the resting potential produced inward but not outward currents. Since no outward currents were seen during the depolarizing step, we assume that potassium currents were substantially reduced or eliminated. In addition to potassium currents, $P$. tetraurelia possess a voltage-sensitive calcium current, a calcium-dependent sodium current, and a magnesium current (Eckert and Brehm 1979; Saimi and Kung 1980; Preston 1990). P. calkinsi possess voltagesensitive calcium and potassium currents (Deitmer and Machemer 1982; Bernal et al. 1991; Bernal and Ehrlich 1992) and may also possess magnesium currents and calcium-dependent sodium and potassium currents. Since the recording medium contained no sodium or magnesium, the inward current was carried predominantly, if not entirely, by calcium ions. Previous work in the laboratory has provided additional evidence that the inward current is a calcium current. For example, the inward current was inhibited, like calcium currents in other cells, by divalent ions, such as $\mathrm{NiCl}_{2}$ and $\mathrm{CdCl}_{2}$ (Bernal and Ehrlich 1992). Moreover, the amplitude of the inward current was increased by $100 \%$ by an increase of calcium concentration in the media from 0.5 to $5.0 \mathrm{mM}$. Deitmer 
A.

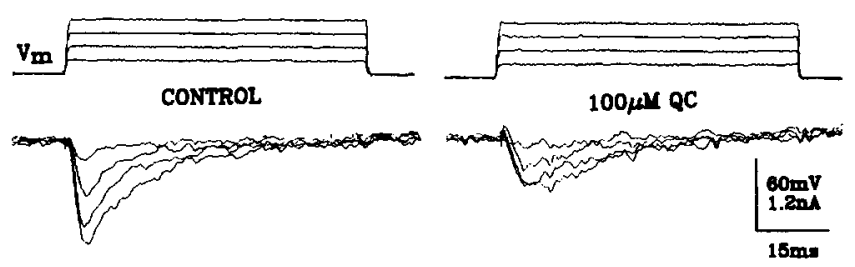

B.

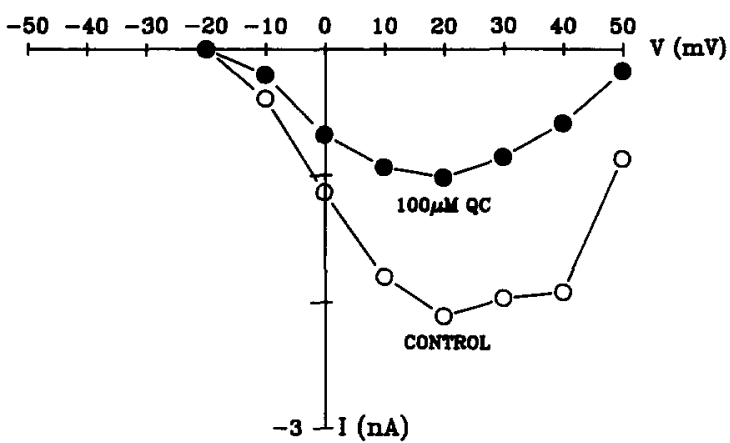

Fig. 8. A At a concentration of $100 \mu M$, quinacrine reduced the peak calcium current by $52 \%$. The cell was held at a membrane potential of $-20 \mathrm{mV}$ and depolarized at $10 \mathrm{mV}$ increments (upper traces) to evoke the calcium currents (lower traces). B Currentvoltage plots of the calcium currents are shown in the presence and absence of $100 \mu M$ quinacrine

and Machemer (1982) have also observed that the inward current recorded from $P$. calkinsi is carried by calcium ions.

The effects of quinacrine were studied in a recording medium containing $15 \mathrm{mM} \mathrm{CaCl}$ since the amplitude of the calcium current was greater in this solution than in a medium containing a lower $\mathrm{CaCl}_{2}$ concentration. Moreover, a $\mathrm{CaCl}_{2}$ concentration of $15 \mathrm{mM}$ was routinely used in other experiments on calcium currents in the laboratory. Since quinacrine's effects on backward swimming were less potent at $15 \mathrm{mM} \mathrm{CaCl}{ }_{2}$ than at lower calcium concentrations, the results reported here may understate the inhibitory actions of quinacrine on calcium currents. Addition of $10 \mu \mathrm{M}$ quinacrine to the preparation decreased the calcium current by $15 \%(n=1), 100 \mu M$ by $51 \pm 2 \%(n=3)$, and $1 \mathrm{mM}$ by $91 \%(n=1)$ (Fig. 8$)$. The drug did not alter the voltage sensitivity or the time to peak of the calcium current. Effects of the drug developed within seconds.

The effects of W-7 were also studied on calcium currents in P. calkinsi (Fig. 9). In $15 \mathrm{mM} \mathrm{CaCl}, 100 \mu M$ W-7 reduced the magnitude of calcium currents at all voltage tested. The maximum inward calcium current was inhibited by $42 \pm 7 \%(n=3)$. At $150 \mu M, \mathrm{~W}-7$ reduced the inward current by $83 \pm 6 \%(n=4)$. These effects developed within seconds.

\section{Toxic effects of $W-7$ and the antimalarial drugs}

W-7 and the antimalarial drugs were toxic to $P$. calkinsi at high concentrations. The drugs all caused similar mor-

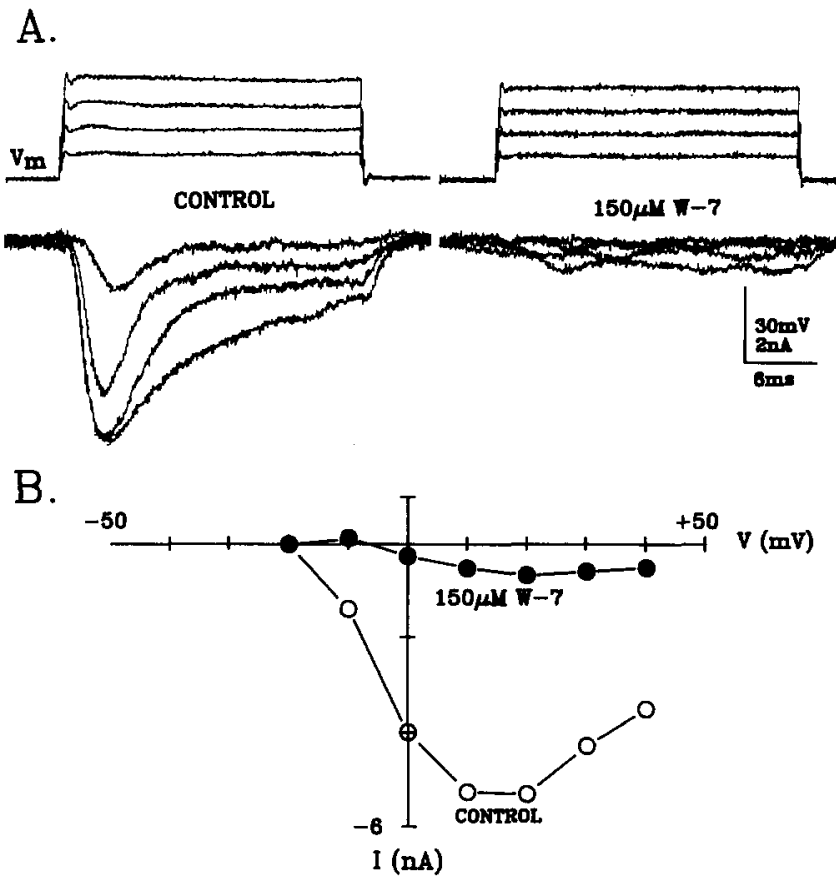

Fig. 9. A At a concentration of $150 \mu M$, W-7 reduced the calcium currents by $88 \%$. The cell was held at a membrane potential of -20 $\mathrm{mV}$ and depolarized at $10 \mathrm{mV}$ increments (upper trace) to evoke the calcium currents (lower trace). B Current-voltage plots of the calcium currents are shown in the presence and absence of $100 \mu M \mathrm{~W}-7$

phological changes. In the presence of high doses of the drugs, the body of the paramecia swelled, the contractile vacuoles became greatly distended, and the cell membrane lysed. In resting solutions containing $1 \mathrm{mM} \mathrm{CaCl}$, these effects developed after $30 \mathrm{~min}$ in the presence of drugs at concentrations of $10 \mu M$ for mefloquine, $100 \mu M$ for primaquine, $150 \mu \mathrm{M}$ for $\mathrm{W}-7$, and $500 \mu \mathrm{M}$ for quinacrine, chloroquine, and quinine. Thus, the paramecia were viable for hours at concentrations of the drugs that produced behavioral effects. Changes in the duration of backward swimming probably did not result from nonspecific toxic effects of the drugs on the paramecia. In recording medium containing $15 \mathrm{mM} \mathrm{CaCl}$, quinacrine produced toxic effects in $30 \mathrm{~min}$ at a concentration of 500 $\mu M$. Effects of quinacrine on the calcium current were seen at $100 \mu M$, a concentration at which paramecia were viable for one hour or more. In recording medium containing $15 \mathrm{mM} \mathrm{CaCl}, 100 \mu M \mathrm{~W}-7$ depressed the calcium current within seconds and produced morphological changes in the paramecia after $20 \mathrm{~min}$.

\section{Discussion}

W-7 and the antimalarial drugs inhibited backward swimming in $P$. calkinsi in low calcium solutions. These effects were seen in 3 different depolarizing solutions including (1) high potassium, sodium solutions, (2) high potassium, sodium-free solutions, and (3) TEA medium that was sodium- and potassium-free and contained potassium channel blockers. The duration of 
potassium-induced backward swimming has been correlated with the activity of voltage-dependent calcium channels in the ciliary membrane in $P$. tetraurelia and $P$. caudatum (Hennessey and Kung 1984; Evans et al. 1987). In $P$. caudatum, calcium channel inhibitors, such as W-7 and neomycin, depress backward swimming at concentrations similar to those necessary to inhibit calcium currents (Hennessey and Kung 1984; Ehrlich et al. 1988; Gustin and Hennessey 1988). The antimalarial drugs share structural similarities with $\mathrm{W}-7$ and, like W-7, reduced the duration of backward swimming in $P$. calkinsi. Thus, these drugs may inhibit the voltage-sensitive calcium channel in paramecia. Doughty has also observed a reduction in potassium-induced backward swimming behavior in P. caudatum by quinine and quin- 2 and has suggested that these effects are mediated by inhibition of calcium channels (Doughty 1988, 1989).

In addition to its effects on calcium currents in paramecia, W-7 is a general calmodulin antagonist (Hidaka et al. 1981). Moreover, in erythrocytes, antimalarial drugs also inhibit calmodulin at micromolar concentrations (Nagai et al. 1987; Nelson et al. 1983; Volpi et al. 1981). It is possible that $P$. calkinsi, like $P$. tetraurelia, possess calcium- and calmodulin-dependent sodium and potassium currents and that the antimalarials inhibit backward swimming by effects on these calcium- and calmodulin-dependent currents (Saimi et al. 1983; Hinrichsen et al. 1986; Preston et al. 1990; Saimi and Ling 1990). Therefore, experiments were designed to determine whether the actions of the antimalarials resulted from effects on sodium and potassium currents.

To rule out the possibility that the drugs depress backward swimming by inhibition of calcium-dependent sodium currents, the actions of the antimalarials were tested in sodium-free medium. Since the drugs inhibited backward swimming in both sodium-containing and sodium-free medium, it is unlikely that the inhibitory actions of the drugs are mediated through effects on calcium-dependent sodium currents.

To determine whether the effects of W-7 and the antimalarials on backward swimming were mediated through potentiation of potassium currents, the actions of the drugs were examined in TEA medium. This medium contained the identical concentrations of potassium channel blockers used to depress potassium currents in the electrophysiological experiments. However, in the electrophysiological experiments, the paramecia were impaled with cesium-filled microelectrodes. Thus, in the voltage clamp but not the behavioral experiments, internal cesium, in addition to external potassium channel blockers, contributed to the depression of potassium currents. Evidence that potassium channels were indeed reduced in the behavioral experiments was provided by the observation that the paramecia swam backward upon transfer to the TEA medium. Backward swimming resulted from depolarization of the paramecia probably because of potassium current inhibition.

W-7 and the antimalarials inhibited backward swimming at similar concentrations in TEA medium as in high potassium medium. These results suggest that the effects of the drugs on backward swimming were not mediated by modulation of sodium or potassium currents but by inhibition of calcium currents. The behavioral experiments do not rule out the possibility that the antimalarials modulate sodium or potassium currents or the possibility that the antimalarials antagonize Paramecium calmodulin. However, the effects of the drugs on backward swimming most likely result from inhibition of the calcium current.

Electrophysiological experiments provide further support that W-7 and quinacrine inhibit backward swimming in $P$. calkinsi by reducing calcium currents. Although quinacrine and W-7 were more effective at reducing backward swimming in $0.05 \mathrm{mM} \mathrm{CaCl} 2$, voltageclamp recordings were performed in a recording medium containing $15 \mathrm{mM} \mathrm{CaCl}{ }_{2}$ because the magnitude of the peak calcium current is greater at the higher calcium concentration. Even though the peak calcium current is greater at $15 \mathrm{mM} \mathrm{CaCl}_{2}$, the duration of backward swimming in $15 \mathrm{mM} \mathrm{CaCl}$ is reduced. This paradox probably results from the fact that the voltage-sensitivity of the calcium current is shifted in the depolarizing direction in high extracellular calcium (Deitmer and Machemer 1982; Bernal and Ehrlich 1992). For a given depolarization in high potassium medium, the calcium current may not be as great in the high as in the low calcium solutions.

In recording medium containing $15 \mathrm{mM} \mathrm{CaCl}, 100$ $\mu M$ quinacrine reduced the inward calcium current by an average of $51 \%$. At the same concentration, quinacrine completely eliminated backward swimming in high potassium solutions. In $15 \mathrm{mM} \mathrm{CaCl} 2,100 \mu M$ W-7 reduced the peak calcium current by $42 \%$ and the duration of backward swimming in high potassium and TEA solutions by 60 and $89 \%$ respectively.

The difference in potency of the drugs in inhibiting backward swimming and calcium currents may indicate that the drugs reduce backward swimming in $P$. calkinsi in part by inhibition of calcium currents and in part by other mechanisms. Alternatively, complete blockade of potassium-induced backward swimming by the drugs may not indicate complete inhibition of the calcium current. In the depolarizing solution containing $15 \mathrm{mM} \mathrm{Ca}-$ $\mathrm{Cl}_{2}, 100 \mu M$ quinacrine completely inhibited backward swimming. However, in the resting solution containing $15 \mathrm{mM} \mathrm{CaCl}, 100 \mu M$ quinacrine did not completely eliminate spontaneous reversals in the direction of forward swimming. Spontaneous reversals result from a transient influx of calcium through calcium channels (Machemer and Sugino 1989). If $100 \mu M$ quinacrine inhibits calcium currents entirely, it should completely block spontaneous reversals. At a lower extracellular $\mathrm{Ca}-$ $\mathrm{Cl}_{2}$ concentration, i.e. $1 \mathrm{mM}, 100 \mu M$ quinacrine eliminates entirely both backward swimming in the depolarizing solution and spontaneous reversals in the resting solution. Since $100 \mu M$ quinacrine does not eliminate spontaneous reversals in $15 \mathrm{mM} \mathrm{CaCl}$, the drug may not completely inhibit the calcium current in this medium.

Calcium currents in P. tetraurelia are not inhibited by organic calcium channel blockers, such as verapamil or the dihydropyridines (Ehrlich et al. 1988). To date, the 
only organic compounds known to inhibit calicum currents in P. tetraurelia and $P$. caudatum are neomycin, other aminoglycosides, W-7, and its derivatives (Hennessey and Kung 1984; Gustin and Hennessey 1988; Ehrlich et al. 1988). Our results suggest that quinacrine may be a more potent inhibitor of calcium currents in $P$. calkinsi than W-7.

The inhibitory effects of the antimalarials on backward swimming were more potent at lower extracellular calcium concentrations. In their studies of $P$. caudatum, Gustin and Hennessey (1988) have also observed that 125 $\mu M$ neomycin inhibits backward swimming and calcium currents in $0.1 \mathrm{mM} \mathrm{CaCl} 2$ but had little effect on backward swimming and calcium currents in $5 \mathrm{mM} \mathrm{CaCl}$. Similarly, Doughty reported that, in P. caudatum, quinine is a more potent inhibitor of backward swimming in low calcium solutions (Doughty 1988).

Gustin and Hennessey (1988) proposed that screening of membrane surface charges may play a role in the inhibitory effects of neomycin and other aminoglycosides on backward swimming and calcium currents in low calcium solutions. In medium containing low calcium concentrations at physiological $\mathrm{pH}$, fixed negative charges on the outer surface of the Paramecium membrane may be largely unscreened. These charges would produce a large negative surface potential between the extracellular bulk phase and the outer surface of the membrane and would decrease the potential difference across the membrane. Screening of these negative charges by calcium or other positively-charged ions would reduce the surface potential and increase the transmembrane potential. These changes in transmembrane potential would affect current flow through ion channels (Eckert and Brehm 1979; Machemer-Röhnisch and Machemer 1989). In our experiments, an increase in $\mathrm{CaCl}_{2}$ concentration from 1 $\mathrm{m} M$ to $15 \mathrm{~m} M$ depressed the duration of backward swimming perhaps by an increase in the transmembrane potential and a decrease in calcium influx through voltage-sensitive calcium channels. Similarly, Gustin and Hennessey (1988) reported that an increase in extracellular calcium reduced the duration of potassium-induced backward swimming in $P$. caudatum.

The antimalarials are all positively-charged at $\mathrm{pH}$ 7.2. Like neomycin and other aminoglycosides, therefore, these drugs may also reduce backward swimming and calcium currents by a charge screening effect. The effects of the aminoglycosides in inhibiting calcium currents increased with charge valence. Neomycin, the most potent aminoglycoside possessed a charge of +6 . In contrast, the antimalarials carry a charge of +1 or +2 at $\mathrm{pH} 7.2$ (Schlesinger et al. 1988; Gustin and Hennessey 1988). Moreover, the effects of neomycin were studied in solutions of low ionic strength where there were few monovalent or divalent cations present to screen membrane surface charges. We have studied the actions of the antimalarials in solutions of higher ionic strength where the charge sceening effects of positively-charged drugs may be reduced. Thus, the role of charge screening in the inhibitory effects of the antimalarials on backward swimming and calcium currents requires further investigation.

Finally, the effects of the antimalarials on backward swimming may be enhanced in low calcium solutions because the binding of the drugs to regulatory molecules involved in backward swimming may be enhanced in low extracellular calcium. For example, the antimalarials may bind to sites involved in the opening of calcium channels or current flow through these channels.

The antimalarials were toxic to the paramecia at high concentrations. Toxic effects may not be mediated through inhibition of calcium currents. For example, the paramecia were viable in $100 \mu M$ quinacrine, a drug concentration that substantially reduced backward swimming and the calcium current. Moreover, the mutant $P$. tetraurelia, Pawn, lacks calcium channels and is viable (Oertel et al. 1977).

However, the effects of W-7 and quinacrine, and perhaps other antimalarials, on calcium currents may explain in part the therapeutic effects of these drugs in the treatment of malaria. Calcium channels in paramecia are pharmacologically different than calcium channels in vertebrate nerve and muscle cells but may be similar to calcium channels in other protozoans (Ehrlich et al. 1988). Malaria is caused by protozoan parasites of the genus Plasmodium. Red blood cells infected with Plasmodium take up at least 7 times more calcium ions than non-infected cells, and over $80 \%$ of the calcium in the red blood cell is concentrated within the parasites (Krungkai and Yuthavong 1983; Leida et al. 1981; Wasserman et al. 1982 ). In addition, invasion of the human malaria parasite, Plasmodium falciparum, into the red blood cell is inhibited by W-7 (Matsumoto et al. 1987; Scheibel et al. 1987). If W-7 and the antimalarials inhibit calcium currents in Plasmodium, the drugs may be effective in blocking invasion of the parasites into the red blood cell and survival of the parasites once within the red cells..

In addition to the effects, described here, of antimalarials on calcium currents in paramecia, quinine and other antimalarial drugs inhibit potassium currents in protozoan and metazoan cells (Saimi and Martinac 1989; Schwarz and Passow 1983). For example, one class of calcium-dependent potassium channels in P. tetraurelia is partially inhibited by quinine and quinidine although at a concentration, $1 \mathrm{mM}$, that is 10 to 100 -fold higher than the concentrations used in these studies (Saimi and Martinac 1989). Furthermore, van Houten (1979) has reported that $100 \mu M$ quinidine, the stereoisomer of quinine, depolarizes and increases the spike frequency of P. tetraurelia. Effects of quinidine may result from inhibition of potassium currents or potentiation of calcium currents. Van Houten observed excitatory effects of quinidine at 1 $\mathrm{mM} \mathrm{CaCl} 2$. In contrast, we observed potent inhibition of backward swimming by the antimalarials at low, 0.05 $\mathrm{mM} \mathrm{CaCl}{ }_{2}$, concentrations. Thus, inhibitory effects of antimalarials on backward swimming and calcium currents may be most prominent at low ambient calcium levels, while additional effects of the antimalarials on calcium and/or potassium currents may be observed at higher extracellular calcium concentrations.

The marine paramecium, $P$. calkinsi, may never encounter low calcium concentrations in its environment. However, intracellular parasites are exposed to the very low calcium levels within the host cell. The antimalarial 
drugs are used to eliminate plasmodia and other intracellular parasites, and their anti-parasitic actions may be enhanced by the low intracellular calcium concentrations. Thus, the increase in potency of these drugs with decreasing ambient calcium concentrations may have important clinical implications.

Acknowledgements. We are grateful to Dr. Barbara E. Ehrlich for support, encouragement, and helpful comments on the manuscript. We would also like to thank Beata Barsi for help with data collection. This work was supported by grants from the Conservation, Food and Health Foundation Inc., the Grass Foundation, and the Biomedical Research Council of the University of Michigan to SRB, NIH grant GM39029 to Barbara E. Ehrlich, and a fellowship from the American Heart Association to JB. We thank HoffmannLa Roche, Inc. for the generous gift of mefloquine.

\section{References}

Barry SR, Bernal J, Ehrlich BE (1988) Quinacrine blocks calcium currents in Paramecium. Biol Bull 175:313

Barry SR, Bernal J, Ehrlich BE (1990) Antimalarial drugs block calcium currents in Paramecium. Biol Bull 179:228

Barry SR, Bernal J, Ehrlich BE (1991) Calcium currents in Paramecium are blocked by antimalarial drugs. Biophys $\mathrm{J}$ 59:277a

Bernal J, Ehrlich BE (1992) Guanine nucleotides modulate calcium currents in marine Paramecium. $\mathrm{J}$ Exp Biol (in press)

Bernal J, Kelsey AM, Ehrlich BE (1991) GTP- $\gamma$-S increases the duration of backward swimming behavior and the calcium action potential in marine Paramecium. J Exp Biol 155:505-518

Deitmer JW, Machemer H (1982) Osmotic tolerance of Ca-dependent excitability in the marine ciliate Paramecium calkinsi. J Exp Biol 97:311-324

Doughty MJ (1988) Action of quinine sulfate on the chemosensory responses of the ciliate, Paramecium caudatum, to inorganic cations. Acta Protozool 27:205-228

Doughty MJ (1989) Differential effects of quin-2 and quin 2-AM on ciliary responses of Paramecium caudatum at moderate calcium levels. Europ J Protistol 25:136-144

Eckert R (1972) Bioelectric control of ciliary activity. Science $176: 473-481$

Eckert R, Brehm P (1979) Ionic mechanisms of excitation in Paramecium. Ann Rev Biophys Bioeng 8:353-383

Ehrlich BE, Jacobson AR, Hinrichsen R, Sayre LM, Forte MA (1988) Paramecium calcium channels are blocked by a family of calmodulin antagonists. Proc Natl Acad Sci USA 85:5718-5722

Evans TC, Hennessey T, Nelson DL (1987) Electrophysiological evidence suggests a defective $\mathrm{Ca}^{2+}$ control mechanism in a new Paramecium mutant. J Membr Biol 98:275-283

Gustin M, Hennessey TM (1988) Neomycin inhibits the calcium current of Paramecium. Biochim Biophys Acta 940:99-104

Hennessey TM, Kung C (1984) An anticalmodulin drug, W-7, inhibits the voltage-dependent calcium current in Paramecium caudatum. J Exp Biol 110:169-181

Hidaka H, Asano M, Tanaka T (1981) Activity-structure relationship of calmodulin antagonists. Molec Pharmocol 20:571-578

Hinrichsen RD, Burgess-Cassler A, Soltvedt BC, Hennessey T, Kung C (1986) Restoration by calmodulin of a $\mathrm{Ca}^{2+}$-dependent $\mathrm{K}^{+}$current missing in a mutant of Paramecium. Science 232:503-506

Krungkrai J, Yuthavong Y (1983) Enhanced $\mathrm{Ca}^{2+}$ uptake by mouse erythrocytes in malarial (Plasmodium berghei) infection. Molec Biochem Parasitol 7:227-235
Leida MN, Mahoney JR, Eaton JW (1981) Intraerythrocytic plasmodial calcium metabolism. Biochem Biophys Res Comm 103:402-406

Machemer H (1989) Cellular behavior modulated by ions: electrophysiological implications. J Protozool 36:463-487

Machemer H, Sugino K (1989) Electrophysiological control of ciliary beating: A basis of motile behaviour in ciliated Protozoa. Comp Biochem Physiol 94A: 365-374

Machemer-Röhnisch S, Machemer H (1989) A Ca paradox : Electric and behavioural responses of Paramecium following changes in external ion concentration. Eur J Protistol 25:45-59

Matsumoto Y, Perry G, Scheibel LW, Aikawa M (1987) Role of calmodulin in Plasmodium falciparum: implications for erythrocyte invasion by the merozoite. Eur J Cell Biol 45:36-43

Nagai F, Ushiyama K, Kano I, Nakagawa A, Nakao T, Nakajima A (1987) Inhibition of calmodulin stimulation of phosphodiesterase and $\mathrm{Ca}^{2+}, \mathrm{Mg}^{2+}$-ATPase activities and shape change of erythrocyte ghosts by chloroquine. Biochem Pharmacol 36:3433-3437

Naitoh Y, Eckert R (1972) Electrophysiology of ciliate protozoa. Exp Physiol Biochem 5:17-31

Nelson GA, Andrews ML, Karnovsky MJ (1983) Control of erythrocyte shape by calmodulin. J Cell Biol 96:730-735

Oertel D, Schein SJ, Kung C (1977) Separation of membrane currents using a Paramecium mutant. Nature 268:120-124

Preston RR (1990) A magnesium current in Paramecium. Science 250:285-287

Preston RR, Wallen-Friedman MA, Saimi Y, Kung C (1990) Calmodulin defects cause the loss of $\mathrm{Ca}^{2+}$-dependent $\mathrm{K}^{+}$currents in two pantophobiac mutants of Paramecium tetraurelia. J Membrane Biol 115:51-60

Saimi Y, Hinrichsen RD, Forte M, Kung C (1983) Mutant analysis shows that the $\mathrm{Ca}^{2+}$-induced $\mathrm{K}^{+}$current shuts off one type of excitation in Paramecium. Proc Natl Acad Sci USA 80:51125116

Saimi Y, Kung C (1980) A Ca-induced Na-current in Paramecium. J Exp Biol 88:305-325

Saimi Y, Ling K-Y (1990) Calmodulin activiation of calcium-dependent sodium channels in excised membrane patches in Paramecium. Science $249: 1441-1444$

Saimi Y, Martinac B (1989) Calcium-dependent potassium channel in Paramecium studied under patch clamp. J Membrane Biol 112:79-89

Saimi Y, Martinac B, Gustin MC, Culbertson MR, Adler J, Kung C (1988) Ion channels in Paramecium, yeast, and Escherichia coli. Cold Spring Harbor Symp Quant Biol 8:667-673

Scheibel LW, Colombani PM, Hess AD, Aikawa M, Atkinson CT, Milhous WK (1987) Calcium and calmodulin antagonists inhibit human malaria parasites (Plasmodium falciparum): implications for drug design. Proc Natl Acad Sci USA 84:7310-7314

Schlesinger PH, Krogstad DJ, Herwaldt BL (1988) Antimalarial agents: Mechanism of action. Antimicrobial agents and chemotherapy 32:793-798

Schwarz W, Passow H (1983) $\mathrm{Ca}^{2+}$-activated $\mathrm{K}^{+}$channels in erythrocytes and excitable cells. Ann Rev Physiol 45:359-374

Van Houten J (1979) Membrane potential changes during chemokinesis in Paramecium. Science 204:1100-1103

Volpi M, Sha'afi RI, Epstein PM, Andrenyak DM, Feinstein MB (1981) Local anesthetics, mepacrine, and propranolol are antagonists of calmodulin. Proc Natl Acad Sci USA 78:795-799

Wasserman M, Alarcon C, Mendoza PM (1982) Effects of $\mathrm{Ca}^{++}$ depletion on the asexual cell cycle of Plasmodium falciparum. Am J Trop Hyg 31:711-717 